\title{
On the onset of transient cavitation in gassy liquids
}

L. Gaete-Garretón, Y. Vargas-Hernández, and R. Vargas-Herrera

Laboratorio de Ultrasonidos, Universidad de Santiago de Chile, Casilla 307, Santiago-2, Chile

J. A. Gallego-Juárez

Instituto de Acústica, Consejo Superior de Investigaciones Científicas, Serrano 144, Madrid 28006, Spain

F. Montoya-Vitini

Instituto de Física Aplicada, Consejo Superior de Investigaciones Científicas, Serrano 144, Madrid 28006, Spain

(Received 17 May 1995; revised 14 February 1996; accepted 18 December 1996)

This paper deals with a numerical and experimental study of ultrasound induced cavitation. On the basis of a computational analysis of Gilmore's equation for the individual bubble dynamics, a slight decrease in the pressure radiated by a single bubble immediately before reaching the transient cavitation regime is predicted. Experiments presented in this paper confirm this observation. Therefore, a new method to detect the inception of transient cavitation is proposed. The numerical analysis of a single bubble behavior, carried out for the different bubble sizes, suggests that two distinct "types" of transient cavitation may occur in a cavitation field in connection with the relative number of bubbles involved in the process. The experiments seem to confirm the theoretical hypothesis. The experimental work consisted mainly of insonifying distilled water, with a gas content of about $75 \%$ of saturation, at $20 \mathrm{kHz}$ and analyzing the resulting acoustic emissions. (C) 1997 Acoustical Society of America. [S0001-4966(97)06304-2]

PACS numbers: 43.25.Yw [MAB]

\section{INTRODUCTION}

One of the most controversial points in relation to cavitation in a gassy liquid is the establishment of clear criteria to determine the real threshold for the onset of transient acoustic cavitation. The analysis of acoustic emissions from the cavitation field could be an adequate way to formulate a modern and objective procedure to detect the inception of cavitation. Nevertheless, the interpretation of the cavitation signals picked up from a liquid where bubbles of different sizes are present is not straightforward. Generally, the emission spectrum has a strong content of harmonics and subharmonics. ${ }^{1,2}$

The idea, which has been suggested several times, is to link the frequency content of the emission with the cavitation state of the liquid. The nonlinear oscillation of a bubble in stable cavitation is a widely studied subject and frequency response curves have been given for different pressure amplitudes. However, when the bubble collapses, no steadystate response can be obtained. Therefore it becomes difficult to establish the relation between the transient cavitation threshold and the emission signals. Some authors have associated the threshold of transient cavitation with a sudden increase of the subharmonic signals. ${ }^{1}$ Nevertheless, the subharmonic cannot be accepted as a clear feature for the onset of transient cavitation because it may also appear in stable cavitation. ${ }^{1}$ In an interesting work, Lauterborn ${ }^{2}$ proposed ultraharmonics of different kinds as the source of the first subharmonic.

In summary, the complex situation described above shows that, even though precise mathematical definitions about dynamical thresholds for cavitation exist, ${ }^{3}$ much work has to be done to establish clear relations between bubble motions and measurable experimental observations. In addition, to study the cavitation field in a gassy liquid, the relations for a single bubble should be extended to a population of differently sized bubbles.

The present paper deals with a numerical and experimental study of cavitation in a gassy liquid with the purpose of defining the onset of transient cavitation and establishing practical methods for detecting the corresponding threshold. The numerical study is based on the Gilmore model for individual bubble dynamics, ${ }^{4}$ where the effects of compressibility of the liquid are included. The experimental study is focused on the collective behavior of different sized bubbles under the action of an acoustic field. Experimental results, in connection with numerical predictions, reveal the existence of two types of transient cavitation in a gassy liquid: a weak and a strong cavitation. A simple method to detect the inception of the strong transient cavitation has been formulated.

\section{EQUATIONS AND NUMERICAL ANALYSIS}

A suitable framework to study acoustic cavitation in water is furnished by the Gilmore equation describing the dynamics of a single cavity under an acoustic field: ${ }^{4}$

$$
\begin{aligned}
R(1 & \left.-\frac{U}{C}\right) \frac{d^{2} R}{d t^{2}}+\frac{3}{2}\left(1-\frac{U}{3 C}\right)\left(\frac{d R}{d t}\right)^{2}-\left(1+\frac{U}{C}\right) H \\
- & \frac{U}{C}\left(1-\frac{U}{C}\right) R \frac{d H}{d R}=0,
\end{aligned}
$$

where $R$ is the bubble radius, $U=d R / d t, C$ is the sound velocity, and $H$ is the specific free enthalpy in the bubble surface. If the process is considered adiabatic, the expression for the free enthalpy will be 


$$
\begin{aligned}
H= & \frac{n}{n-1} \frac{A^{1 / n}}{\rho_{0}}\left\{\left[\left(P_{0} 2 \frac{s}{R_{0}}\right)\left(\frac{R_{0}}{R}\right)^{3 \gamma}-2 \frac{s}{R}+B\right]^{(n-1) / n}\right. \\
& \left.-\left[P_{0}-P_{n} \sin (\omega t)+B\right]\right\}
\end{aligned}
$$

and the sound velocity,

$$
C=\left[C_{0}+(n-1) H\right]^{1 / 2},
$$

where $A, B$, and $n$ are constants (for water $A=3001$ atm, $B=3000 \mathrm{~atm}, n=7), P_{n}$ is the pressure amplitude of the applied acoustic field, $P_{0}$ is the static pressure, $R_{0}$ is the equilibrium bubble radius, $\rho_{0}$ is the equilibrium density of the liquid, $C_{0}$ is the sound velocity in the unperturbed liquid, $\omega$ is the angular frequency, $\sigma$ is the surface tension, and $\gamma$ is the ratio of the specific heats. These equations take account of the compressibility of the medium, but viscosity and mass transfer are not included.

The numerical solution of Eqs. (1), (2), and (3), using the Runge-Kutta fourth-order algorithm, will determine the motion of the bubble and the conditions under which transient cavities will be produced. The values used for the various parameters are: $\sigma=75 \mathrm{dyn} / \mathrm{cm}^{2}, \gamma=4 / 3, \omega=2 \pi \times 20000$ $\mathrm{rad} / \mathrm{s}, C_{0}=1550 \mathrm{~m} / \mathrm{s}$, and $P_{0}=1.013 \times 10^{5} \mathrm{~Pa}$. A typical calculation lasts for about seven acoustic periods.

The study of cavitation in a gassy liquid containing initially a wide distribution of small bubbles that serve as cavitation nuclei will be made considering the bubble resonance radius as a critical parameter. The resonance radius $R_{r}$, determined by the acoustic field frequency and the liquid characteristics, can be calculated with the following expression obtained from the linearized equations: ${ }^{4}$

$$
\rho \omega_{r}^{2} R_{r}^{2}=3 \gamma\left(P_{0}+2 \frac{\sigma}{R_{r}}\right)-\frac{\sigma}{R r},
$$

where $\omega$ is the frequency of the applied sound field, and $\rho$ the liquid density. As an example, for an applied acoustic field of $20 \mathrm{kHz}$ in water at $20{ }^{\circ} \mathrm{C}$ the resonance radius will be $0.016 \mathrm{~cm}$.

Some results from the numerical solution of Eqs. (1)(3) are shown in Fig. 1. The radiated sound pressure near the cavity wall for different bubble sizes was computed by using the equation of state for water in the form $P=A\left(\rho / \rho_{0}\right)^{n}-B$, and following the analysis presented in Ref. 4 . It is displayed as a function of the applied sound pressure at a frequency of $20 \mathrm{kHz}$. It can be observed that the slopes of the curves suddenly change at a certain point, tending to infinity. The applied acoustic pressure which produces such a change for each bubble size can be identified as the transient cavitation threshold for the corresponding bubble. In fact, if we calculate the bubble wall velocity at this point, values of the order or even higher than sound velocity are obtained, which represents another indication for transient cavitation. ${ }^{5}$ As an example, Fig. 2 shows the wall velocity of a bubble of radius $0.004 \mathrm{~cm}$. It is apparent that for an applied acoustic field of about 0.9 atm the wall velocity suddenly increases and tends to infinity. In such conditions, the bubble collapses and a shock wave will be formed. ${ }^{1}$ It should be noted that immediately before the cavitation threshold pressure is reached, a

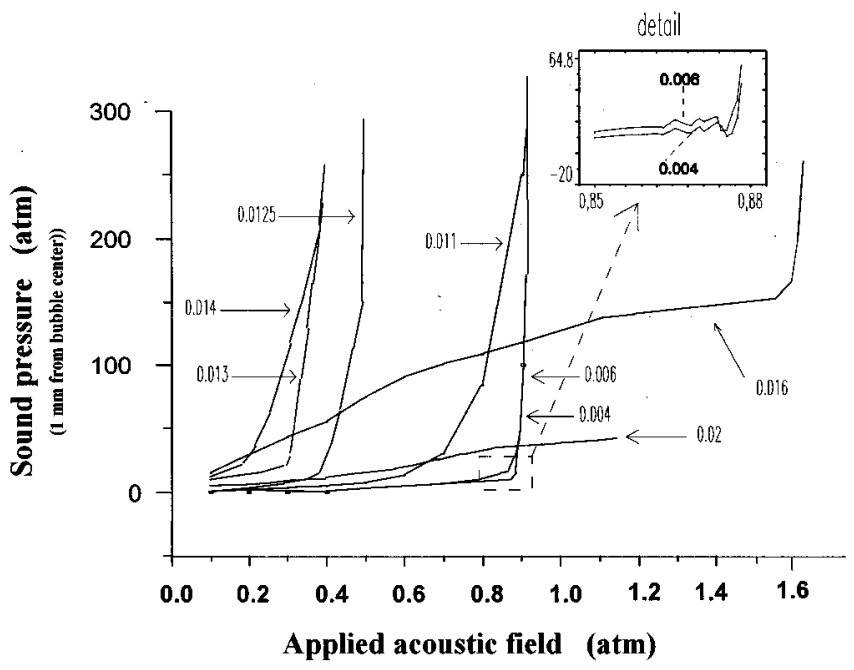

FIG. 1. Sound pressure near the bubble wall versus applied acoustic pressure at $20 \mathrm{kHz}$ (from the Gilmore equation). The numbers attached to each curve represent the equilibrium bubble radii in $\mathrm{cm}$.

small drop in the emitted sound pressure is observed. This effect is shown in the detail of Fig. 1 for two bubbles of 0.004 and $0.006 \mathrm{~cm}$ in radii, respectively.

Figure 3 presents the thresholds computed for single bubbles of radii below and above the resonance radius. The criterion used to produce this figure was to take the value at which the bubble wall velocity reaches the sound velocity value in the unperturbed liquid for the pressure threshold. It can be observed that bubbles with radii within the range $0.001-0.012 \mathrm{~cm}$ exhibit a similar threshold pressure for the onset of transient cavitation (ca. $0.9 \mathrm{~atm}$ ). Therefore the population of bubbles included within this wide size range could be the cause of a great cavitation activity in a gassy liquid as they will act as nuclei to produce a simultaneous collective transient cavitation when the threshold pressure is reached. In this paper this type of cavitation is called "strong transient cavitation." There is another characteristic group of bubbles, with radii just below the resonance radius, where the threshold pressure is different for each size of bubble and generally of a lower value than the threshold for the so-called

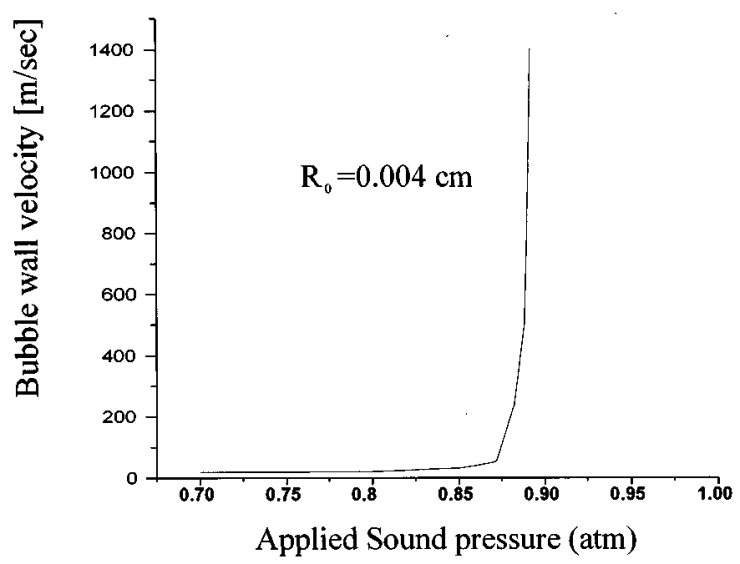

FIG. 2. Bubble wall velocity as a function of applied acoustic field for a bubble of $R_{0}=0.004 \mathrm{~cm}$ (from the Gilmore equation). 


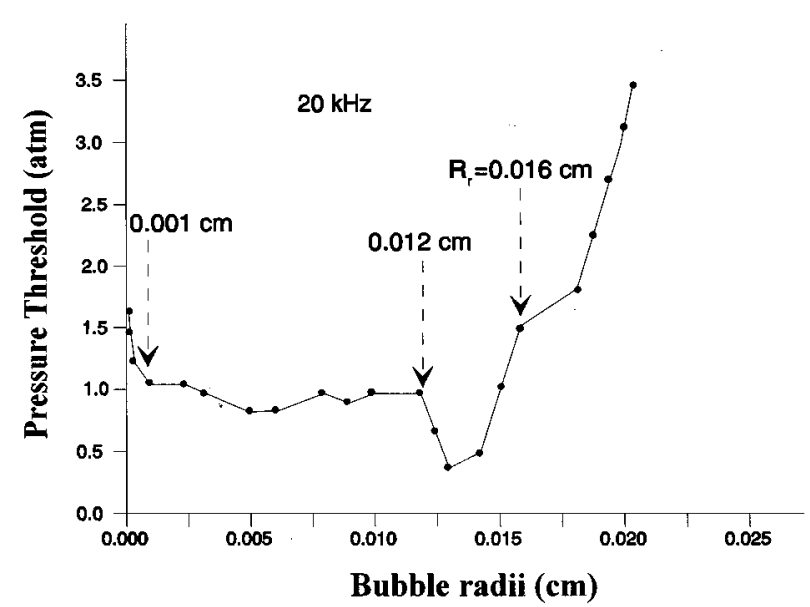

FIG. 3. Cavitation thresholds computed for single bubbles of different radii.

"strong cavitation." The bubble radii of this group for the example here considered, range between 0.012 and 0.016 $\mathrm{cm}$. At the lower threshold pressure (about $0.4 \mathrm{~atm}$ ) the number of collapsing bubbles is small and increases if the applied pressure rises. Therefore, it seems evident that in a real liquid, where a relatively homogeneous distribution of bubbles of radii between $10^{-3}$ and $2 \times 10^{-2} \mathrm{~cm}$, can be expected, ${ }^{6}$ the cavitation activity produced by the collapsing bubbles within the $0.012-0.016 \mathrm{~cm}$ range is generally smaller than that produced by the collapsing bubbles within the $0.001-0.012 \mathrm{~cm}$ range. As a consequence, we believe we have identified a new kind of cavitation regime, the "weak transient cavitation" in a liquid. The threshold pressure for this type of cavitation is lower than that required for "strong cavitation." Finally, there is a third group of bubbles, with radii above the resonance radius, which need a much higher applied acoustic pressure to produce cavitation.

In summary, in a gassy liquid with a wide bubble size distribution, two main cavitation processes are expected. At relatively low pressures (in the example here reported between approximately 0.3 and 0.8 atm) a weak transient cavitation will be produced. At a higher level (about 0.9 atm in this case) strong cavitation will occur because of the large number of transient cavities involved. The collective nature of this latter process allows the threshold to be well-defined. In this next section, experimental evidence of these events will be presented.

\section{EXPERIMENTAL WORK}

The experimental work consisted in the insonification at $20 \mathrm{kHz}$ of distilled water with a certain air content and in measuring and analyzing the acoustic emission from the cavitation field. The experimental setup is shown schematically in Fig. 4. A piezoelectric piston source $30 \mathrm{~mm}$ in diameter radiates at $20 \mathrm{kHz}$ in an anechoic cylindrical container $(25 \mathrm{~cm}$ in diameter and $26 \mathrm{~cm}$ in height) filled with distilled gassy water (air content of about $75 \%$ saturated). The emission signal was picked up by a probe specially designed to work under high acoustic pressures in a cavitation environment. ${ }^{7}$ The applied power was controlled by an electronic system that keeps the output power constant during the

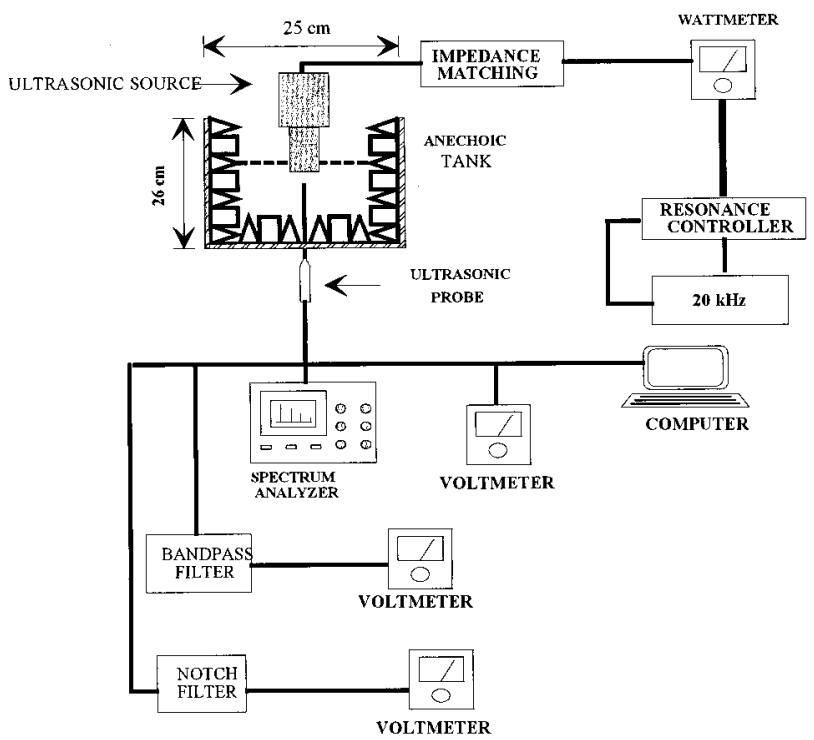

FIG. 4. Experimental setup to study the emission from a cavitation field.

experiment. ${ }^{8}$ The received signals were analyzed by using a superheterodyne spectrum analyzer, 40-Mhz bandwidth and 100-dB dynamic range. In the present measurements the operating band used was $10-200 \mathrm{kHz}$ with a frequency resolution of $10 \mathrm{~Hz}$ and an amplitude accuracy of $0.2 \mathrm{~dB}$.

Figure 5 shows the rms values of the total pressure received with the probe and measured with the voltmeter, versus the fundamental component of the same signal. It can be observed that for a pressure value of about 0.86 atm, the amplitude of the received signal shows a drop just before the onset of strong cavitation. Therefore it seems advisable to use this point, where the change of the slope of the curve is very clear, as the indication of the onset of cavitation. To that purpose, the excitation signal was filtered out from the received signal by introducing a notch-filter (6-dB cutoff frequency $2 \mathrm{kHz}$ ) (Fig. 4). As is well-known a notch-filter is an electronic device used to eliminate a narrow band in a wide-

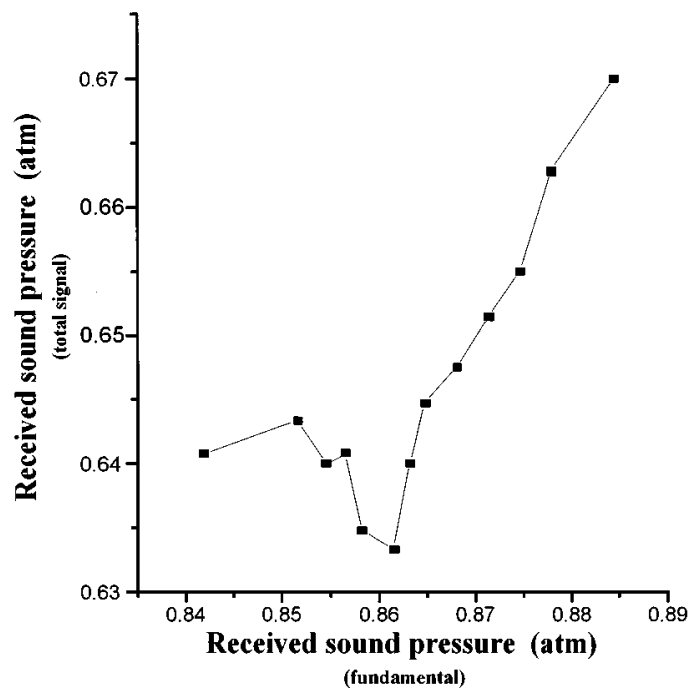

FIG. 5. Total sound pressure received from the cavitation field as a function of the fundamental signal. 


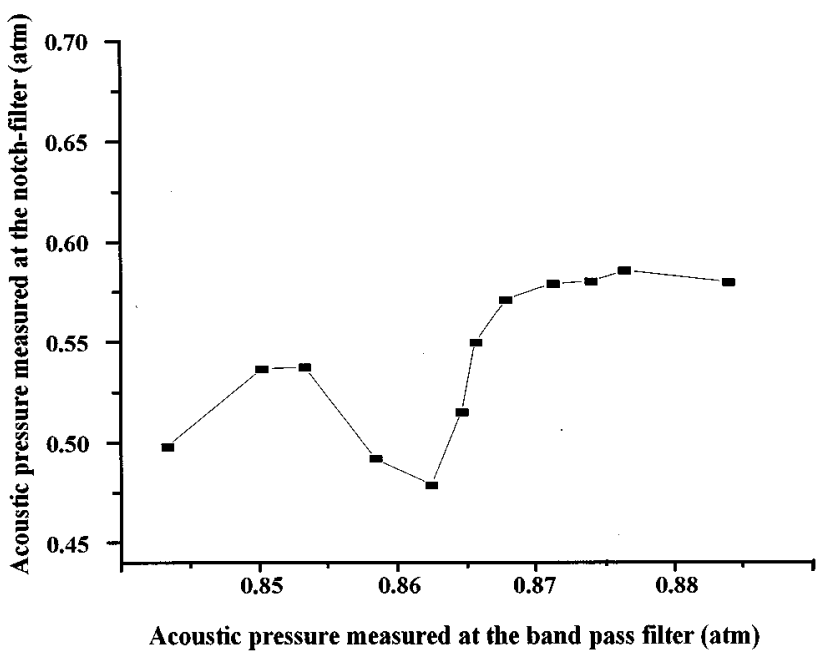

FIG. 6. Acoustic pressure measured at the notch-filter versus acoustic pressure measured at the bandpass filter.

band signal. In such a way the driving signal was removed from the emission spectrum. The fundamental component of the received signal was measured by using a bandpass filter (6-dB cutoff frequency $1 \mathrm{kHz}$ ) (Fig. 4). Representing the signal amplitude from the notch-filter output as a function of the signal amplitude from the bandpass filter output, the curve of Fig. 6 was obtained. It is evident that the curve drop is emphasized by this measuring procedure.

The onset of weak cavitation cannot be so well-defined because it depends on the bubble size and consequently is also linked to the collapse of a small number of cavities. Experimentally we have detected the onset of this cavitation by exposing a thin aluminum film to an acoustic field of about 0.34 atm. The photograph of Fig. 7(b) shows the weak erosion effect produced. The corresponding emission spectrum does not contain subharmonic components. A similar test carried out at an applied acoustic pressure of about 1 atm shows the strong erosion effect produced on the metallic film exposed to the cavitation field [Fig. 7(c)]. In this case, the received spectrum clearly shows subharmonic content.

\section{DISCUSSION AND CONCLUSIONS}

The results obtained numerically from the Gilmore's equation for a single cavity seem to be a good basis for explaining the production of acoustic cavitation in a gassy liquid. Nevertheless, there are a few points which still are not well-explained. For instance, the decrease that the signal amplitude received from the cavitation field suffers just before the onset of the strong cavitation. A possible interpretation of this behavior can be made in terms of the numerical results of the Gilmore equation. In the curves of Fig. 1, it was observed that the sound pressure emitted for each different size of bubble drops slightly just before reaching the threshold point (see the detail in Fig. 1). Therefore, as there are a large number of bubbles that show this effect for about the same level of acoustic pressure, the collective behavior could result in a more pronounced drop in the slope of the curve. The physical explanation of this effect is not so apparent, though
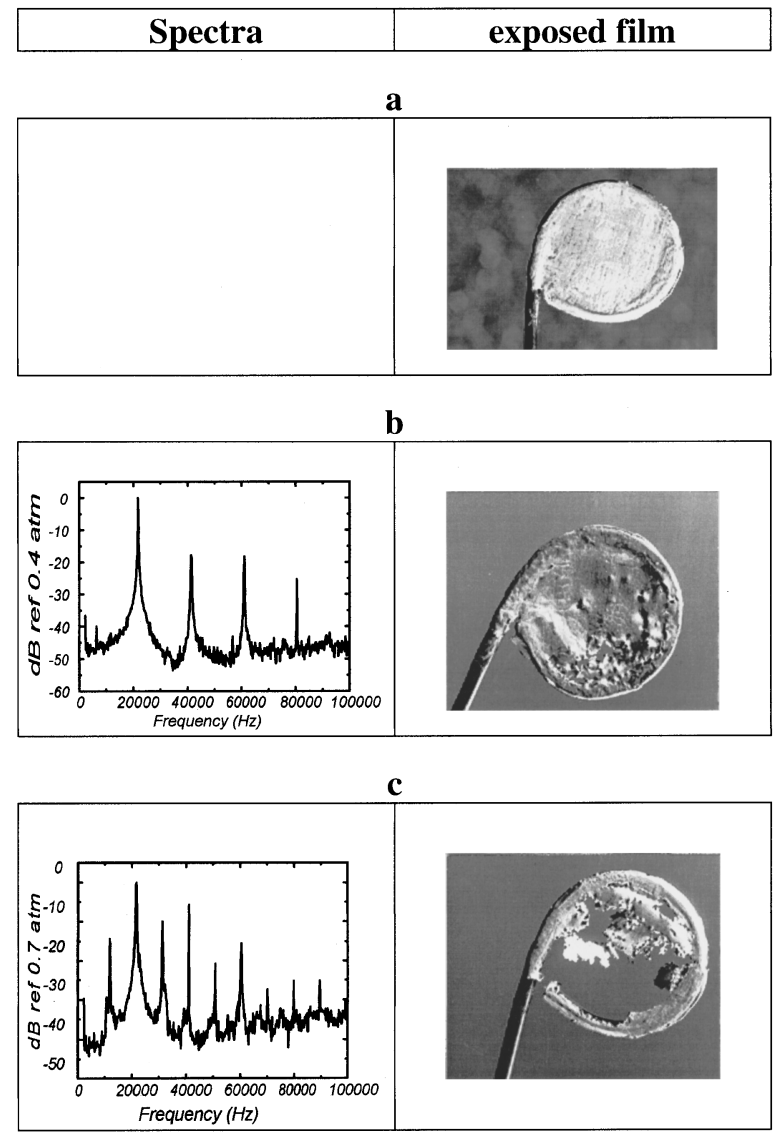

FIG. 7. Emission spectra from weak (b) and strong (c) transient cavitation fields and photographs of the corresponding erosion effects on aluminum films.

it could be attributed to an increase of the dissipative mechanism when the bubble motion approaches that of the transient cavity.

In conclusion, the present numerical and experimental study of transient cavitation in gassy liquids has shown the existence of two types of transient cavitation: Strong cavitation, which can be attributed to the collective behavior of a large number of bubbles, and weak cavitation, which occurs at lower acoustic pressures and seems to be produced by a restricted number of cavities.

\section{ACKNOWLEDGMENT}

We acknowledge the financial support from the European Commission, project CI1*-CT92-0032, FONDECYT project 105-92, and CICYT project, TAP93-230.

${ }^{1}$ E. A. Neppiras, “Acoustic Cavitation,” Phys. Rep. 16, 161-251 (1980).

${ }^{2} \mathrm{~W}$. Lauterborn, "Numerical investigation of nonlinear oscillation of gas bubbles in liquids," J. Acoust. Soc. Am. 38, 283-293 (1976).

${ }^{3}$ H. G. Flynn, "Cavitation dynamics. II. Free pulsation and models for cavitation bubbles," J. Acoust. Soc. Am. 58, 1160-1170 (1975).

${ }^{4}$ V. A. Akulichev, "Pulsation of cavitation voids" in High Intensity Ultrasonic Fields, edited by L. D. Rozenberg (Plenum, New York, 1971), pp. 203-259.

${ }^{5}$ P. N. Vaughan and S. Leeman, “Acoustic cavitation revisited," Acustica 69, 109-119 (1989).

${ }^{6}$ M. G. Sirotyuk, "Experimental investigations of ultrasonic cavitation," in 
High Intensity Ultrasonic Fields, edited by L. D. Rozenberg (Plenum, New York, 1971), pp. 263-343.

${ }^{7}$ L. Gaete-Garretón, Y. Vargas Hernández, S. Pino Dubreuil, and F. Montoya-Vitini, "Ultrasonic detectors for high-intensity acoustic fields,"
Sens. Actuators A 37-38, 410-414 (1993).

${ }^{8}$ A. Ramos Fernández, F. Montoya Vitini, and J. A. Gallego-Juárez, “Automatic system for dynamic control of resonance in high power and high $Q$ ultrasonic transducers," Ultrasonics 23, 151-156 (1985). 